\title{
A Descriptive Study of the Implementation of Remote Occupational Rehabilitation Services Due to the COVID-19 Pandemic Within a Workers' Compensation Context
}

\author{
Douglas P. Gross ${ }^{1}$ (1) $\cdot$ Alexander Asante ${ }^{2} \cdot$ Joanne Pawluk $^{3} \cdot$ Riikka Niemeläinen $^{3}$
}

Accepted: 17 October 2020 / Published online: 28 October 2020

๑) Springer Science+Business Media, LLC, part of Springer Nature 2020

\begin{abstract}
Purpose The Coronavirus Disease (COVID-19) pandemic resulted in dramatic changes to avoid virus spread. In Canada, following provincial legislation the Workers' Compensation Board of Alberta (WCB-Alberta) stopped in-person rehabilitation services on March 23, 2020. On April 1, training began on remote service delivery using videoconferencing or telerehabilitation, which started April 3. We studied WCB-Alberta's transition to remote rehabilitation service delivery. Methods A population-based descriptive study was conducted, with data extracted from the WCB-Alberta database. This included clinical data from rehabilitation providers. We included workers completing services between January 1 and May 31, 2020. We statistically examined differences before and after the transition to remote services. Results The dataset included 4,516 individuals with work-related injuries. The mean number of work assessments per week pre-COVID was 244.6 (SD 83.5), which reduced to 135.9 (SD 74.5). Workers undergoing remote assessments were significantly more likely to work in health care or trades, did not require an interpreter, and were less likely to be working or judged as ready to return to work. Number of completed rehabilitation programs also reduced from 125.6 to 40.8 per week, with most (67.1\%) remote programs being functional restoration. Few adverse effects were observed. Conclusions We describe the transition to completely remote delivery of occupational rehabilitation due to COVID-19 physical distancing restrictions in one Canadian compensation jurisdiction. It appears the use of remote services was successful but proceeded cautiously, with fewer complex cases being referred for assessment or rehabilitation. Further research examining longer-term work outcomes and stakeholder perceptions is needed.
\end{abstract}

Keywords Rehabilitation · Musculoskeletal diseases · Compensation and redress · COVID-19 · Telehealth

\section{Introduction}

In early 2020, the coronavirus disease (COVID-19) [1] pandemic resulted in dramatic changes to delivery of health care services world-wide to avoid transmission and spread of the novel corona virus [2]. Many jurisdictions transitioned to remote delivery of services using telehealth or videoconferencing technology [3]. Preliminary evidence has shown that

Douglas P. Gross

dgross@ualberta.ca

1 Department of Physical Therapy, University of Alberta, 2-50 Corbett Hall, Edmonton, AB T6G 2G4, Canada

2 Suncor Energy, Calgary, Canada

3 Workers' Compensation Board of Alberta, Edmonton, Canada telehealth/ telerehabilitation and videoconferencing as used by physical therapists has promise for treating individuals with musculoskeletal conditions [4,5]. However, little is known about these services in the context of occupational rehabilitation where physical in-person testing and participation in work-related functional activities are the norm. As physical distancing restrictions may last for several months or until a vaccine is available, sharing experiences with remote service delivery could inform other jurisdictions considering this option.

In Alberta, Canada, under provincial legislation in March 2020, all in-person visits to rehabilitation professionals were stopped with the exception of urgent or emergency cases. On March 23, 2020, the Workers' Compensation Board of Alberta (WCB-Alberta) stopped offering all inperson rehabilitation services to injured workers. However, in early April 2020 WCB-Alberta transitioned to remote 
delivery of assessment and treatment services via telephone or videoconferencing.

Previous research conducted in Alberta had evaluated conversational assessment and rehabilitation strategies. The Functional Interview Tool (FIT) for assessing work ability had been developed [6] and evaluated [7, 8] in a randomized controlled trial examining the value of performance-based functional capacity evaluation (FCE). Findings indicated the FIT led to comparable return to work outcomes as FCE, with lower risk of pain exacerbation during rehabilitation. Since the FIT incorporated principles of motivational interviewing [9], subsequently the effectiveness of motivational interviewing as an adjunct to routine occupational rehabilitation was examined [10, 11]. Incorporating motivational interviewing into functional restoration programs led to higher return-to-work rates at program discharge in non-job attached workers and more sustained return to work over a follow-up year.

Experience with these clinical trials allowed WCBAlberta to rapidly transition to remote delivery of assessment and treatment using the tools and resources previously developed. On April 1, 2020, the first training sessions were offered to rehabilitation professionals on how to conduct the FIT protocol over videoconferencing. On April 3, 2020, the first injured workers were assessed using the FIT to determine work-related functional ability, readiness to return to work, and need for further rehabilitation services. All other assessment and rehabilitation programs (except neuropsychological assessments) also transitioned to remote delivery in early April 2020.

The objective of this research was to describe WCBAlberta's transition to remote delivery of occupational rehabilitation services in response to the COVID-19 pandemic. We examined the characteristics of injured workers completing work assessments or rehabilitation programs in the first three months of the year (before COVID-19) and compared them to workers completing work assessments or rehabilitation programs after transition to remote services (after April 3, 2020).

\section{Methods}

\section{Design}

A population-based descriptive study was conducted, with data extracted from the WCB-Alberta's provincial database. This database contains information on thousands of injured workers and is augmented by clinical data from contracted rehabilitation providers in the province. These providers complete reports at time of injured worker's assessment, as well as admission and discharge from rehabilitation. We used data collected on workers completing work assessment or rehabilitation programs between January 1 and May 31, 2020. Ethical approval was obtained from the University of Alberta's Health Research Ethics Board.

\section{Population}

Province-wide data were available on all injured workers with open WCB-Alberta claims who completed work assessment or rehabilitation during the study period. Based on a soft tissue injury continuum of care model, injured workers are referred for work assessment when they have met or surpassed expected injury healing times (i.e. 4-8 weeks) and have plateaued with medical interventions, yet report ongoing difficulties related to their compensable condition. Assessing clinicians interpret assessment findings and injured workers are triaged to what is deemed the most appropriate option (usually return to work at some level or a rehabilitation program).

\section{Study Procedures}

This study was limited to archived clinical and administrative data of the WCB-Alberta. No injured workers were directly recruited. Within Alberta, WCB reports are electronic and data is thus automatically entered into WCBAlberta databases. Characteristics of injured workers were linked with routinely collected program evaluation data.

\section{Measures}

We extracted data on all injured workers completing work assessment or occupational rehabilitation programs across the province. Specific measures extracted included worker characteristics, details of the type of work assessment or rehabilitation program delivered, and clinical recommendations following the assessment or discharge from rehabilitation.

\section{Worker Characteristics}

This included age, sex, injury duration in days, primary diagnosis, injured body part, whether the worker experienced a comorbid injury or condition, National Occupational Classification code, employment status (i.e., job attachment status), current work status, availability of modified work, and scores on self-reported outcome measures completed at the time of assessment. These included the Pain Disability Index (PDI) [12], pain Visual Analogue Scale (VAS, $100 \mathrm{~mm}$ ) [13], the 36-Item Medical Outcome Study Short Form Health Survey (SF-36) [14]. Depending on part of body affected, workers also completed the short-form Disabilities of the Arm Shoulder and Hand (QuickDASH) questionnaire [15], Lower Extremity Functional Scale (LEFS) 
[16], or the Orebro Musculoskeletal Pain Questionnaire (OMPQ) $[17,18]$.

\section{Type of Work Assessment}

Clinicians typically use the WorkWell Functional Capacity Evaluation (FCE) within the WCB-Alberta jurisdiction [19]. This is a performance-based functional assessment protocol that is conducted as either a basic (1-day) assessment or a more comprehensive (2-day) protocol. Basic assessments are reserved for more straight-forward cases and are typically requested when claim owners suspect further rehabilitation is needed, while comprehensive assessments are completed in more chronic, complex situations (i.e., are considering claim closure and likelihood of ongoing work restrictions). After March 23, 2020, all assessments were done using videoconferencing or telephone and the FIT protocol described earlier.

The FIT uses a semi-structured interview format, incorporating principles of self-efficacy theory and motivational interviewing. In head-to-head comparisons, the FIT has been shown to result in similar return to work outcomes as traditional performance-based FCE [8]. Training on how to conduct the FIT via remote delivery was provided via synchronous online training by two of the authors (JP, AA). The telehealth equipment and software used for remote work assessment was at the discretion of the rehabilitation providers, but most often involved Cisco WebEx (Milpitas, California) or Microsoft Teams (Redmond, Washington).

\section{Type of Rehabilitation}

After work assessment, workers who are not judged as ready to return to work are typically recommended for further rehabilitation. Five main types of occupational rehabilitation programs are offered in the jurisdiction [20]. This includes multidisciplinary functional restoration, workplace-based interventions (typically in conjunction with a functional restoration program), complex biopsychosocial chronic pain treatment, brain injury and psychological injury programs (which may be focused on cumulative or traumatic psychological injury).

After the COVID-19 pandemic, remote rehabilitation programs included one-on-one services with a physical, occupational, or exercise therapist through telehealth or over the phone. These programs could also include emailed educational material, individual counselling and problem solving, and/or group classes depending on the needs of the worker. Injured workers were provided with individualized rehabilitation programs focusing on their compensable injury and critical job demands. While there were few changes to the exercises provided by physical and exercise therapists (i.e., therapeutic exercise, general aerobic fitness, strengthening, and flexibility exercise), functional restoration programs included creative options for work simulation and manual handling activities. This typically included identifying items of various weights around an individual's house and gradually progressing load by adding items to a suitable box or other household container that could be used as a lifting unit.

\section{Outcome Recommendations}

After work assessment, clinicians make recommendations and determinations regarding work ability for each injured worker. Options include return to full pre-accident work duties (return-to-work if employed or fit-to-work if unemployed), able to participate in modified work (duties, hours, or both), or not able to participate in any work. If unable to return to full work duties, the clinicians comment on the nature of the work restrictions as either temporary or permanent, as well as the anticipated duration of work restrictions in weeks if temporary. Lastly, the clinicians make recommendations regarding further interventions needed. Options include no further treatment needed, treatment by a community provider (i.e. physical or occupational therapist, psychologist, or chiropractor), multidisciplinary occupational rehabilitation, or further medical services needed. Outcomes following rehabilitation are similar, but focus on work ability rather than future treatment needed.

\section{Adverse Events}

We formally and informally monitored the number and type of adverse events or unwanted consequences from remote service delivery. In the jurisdiction, adverse effects are monitored via stakeholder feedback from claim owners, treatment providers, and injured workers. Feedback is solicited through satisfaction surveys or unsolicited from telephone or email contact. We monitored for complaints about telerehabilitation or any other atypical outcomes. In addition to stakeholder feedback, we monitored trends and themes arising from appeals and claim-decision fairness reviews.

\section{Statistical Analysis}

Initially, all data records were reviewed to determine if any data issues such as missing data, outliers or out of range values existed. Such occurrences were infrequent, however, substantial missing data existed for the self-report questionnaires. Descriptive statistics were then calculated including means and standard deviations for continuous variables, modes and percentages for categorical variables. We compared differences between workers completing assessment or rehabilitation before and after the transition to remote services using independent $t$-tests for continous variables and chi square test for categorical variables. Since so few 
comprehensive work assessments were conducted remotely, we also statistically compared the clinical characteristics and outcome recommendations of workers undergoing basic work assessment before and after the transition to remote work assessment. All analyses were completed in IBM SPSS v25 (Armonk, New York). An alpha of 0.05 was used to judge statistical significance.

\section{Results}

\section{Characteristics of Workers Undergoing Work Assessment}

The dataset included 4,516 individuals with open workers' compensation claims for a wide variety of work-related injuries. Worker characteristics according to the type of work assessment undertaken are shown in Table 1 . The majority of workers were employed (81.3\%) and working in trades and transport occupations (42.1\%) (See Table 1). Average age was 45.9 years and median injury duration was 112 days.

\section{Effect of COVID-19 Restrictions on Volume of Services}

Figure 1 shows the number of work assessments completed within the jurisdiction over the timeframe of the study. The mean number of assessments per week conducted before the lock-down of March 23 was 244.6 (SD 83.5), while the mean number after transitioning to the FIT was 135.9 (SD 74.5) per week. Of note are the very limited number of comprehensive work assessments conducted after the transition to remote assessments, indicative of less complicated cases undergoing assessment. The mean number of workers completing rehabilitation programs per week before March 23 was 125.6 (SD 45.1), while the mean number after transitioning to remote rehabilitation was 45.1 (SD 22.3).

\section{Differences Between Workers Assessed Before and After COVID-19 Restrictions}

Table 1 provides a detailed comparison between workers assessed before and after the transition to remote work assessments. After the start of the COVID-19 pandemic, very few $(\mathrm{n}=18,1.5 \%)$ comprehensive assessments were conducted. Workers assessed using remote assessments were significantly more likely to work in Health (12.3\% versus $9.9 \%$ ) or Trades and Transport (44.1\% versus $41.4 \%$ ) and less likely to work in Sales and Service (16.5\% versus $19.7 \%$ ) or Business, Finance or Administration (4.7\% versus 6.7\%) industries. They also had significantly higher paying jobs (mean annual salary of $\$ 51,500 \mathrm{CDN}$ versus $\$ 48,000 \mathrm{CDN}$ ), and were less likely to require an interpreter during assessment (1.6\% versus $2.8 \%)$.
They were also significantly less likely to be employed (76.5\% versus $83.1 \%$ ), currently working ( $37.9 \%$ versus $52.5 \%$ ), or have modified work available (46.0\% versus $51.9 \%$ ).

\section{Work Assessment Outcomes Before and After COVID-19 Restrictions}

Table 2 provides a comparison of basic work assessment outcomes recommended by clinicians before and after the transition to remote assessments. Workers assessed using remote assessments were significantly less likely to be judged as ready to return to pre-accident functional work levels (3.4\% versus $6.8 \%$ ) and more likely to be recommended modified work duties $(69.6 \%$ versus $60.5 \%)$. They were also more likely to be given permanent restrictions ( $7.5 \%$ versus $1.1 \%$ for return to work restrictions and $13.3 \%$ versus $6.2 \%$ for fit to work restrictions). When recommended, temporary work restrictions given to workers assessed using remote assessment were for a significantly shorter duration in the case of return to work (3.5 weeks versus 4.4 ) but longer duration in the case of fit to work restrictions (4.6 weeks versus 3.8). Workers assessed remotely were more likely to have no rehabilitation programs recommended $(9.3 \%$ versus $6.1 \%)$. When rehabilitation was recommended, it was more likely to be a single service community provider $(28.1 \%$ versus $23.7 \%)$ rather than a multidisciplinary program $(53.4 \%$ versus $59.1 \%)$.

\section{Self-Reported Outcome Measure Scores Before and After COVID-19 Restrictions}

Table 3 shows clinical characteristics of workers undergoing basic work assessment and completing the various self-reported pain intensity, disability, and health-related quality of life measures $(n=2,356)$. Average pain intensity (49.2/100 mm on the VAS) and disability (47.8/100 on the PDI) were moderate. Scores on most measures were comparable and differences did not reach clinically meaningful levels. However, workers undergoing remote assessments reported statistically significantly higher pain intensity (51.2 versus $48.4 \mathrm{~mm}$ ), worse 'role physical' function on the SF-36 (28.5 versus 31.0), and worse function on the LEFS (40.3 versus 44.6) and QuickDASH work module (60.0 versus 49.1). Scores on the social function (52.7 versus 50.1) and role emotional (54.0 versus 50.4) domains of the SF-36 were significantly better among workers undergoing remote assessments.

\section{Characteristics of Workers Completing Occupational Rehabilitation}

Over the study period, 1,755 individuals completed a rehabilitation program. Characteristics of workers undergoing 
Table 1 Characteristics of injured workers undergoing work assessment before and after transitioning to remote assessments

\begin{tabular}{|c|c|c|c|c|}
\hline & $\begin{array}{l}\text { All workers } \\
\mathrm{n}=4,516\end{array}$ & $\begin{array}{l}\text { Jan. 1-Mar. } 23 \\
\text { Regular FCE } \\
\mathrm{n}=3,293\end{array}$ & $\begin{array}{l}\text { April 3-May } 31 \\
\text { Remote FIT } \\
\mathrm{n}=1,223\end{array}$ & $\mathrm{p}$ value \\
\hline & \multicolumn{4}{|c|}{ Values represent mean (standard deviation) or $\mathrm{n}(\%)$} \\
\hline Age (years) & $45.9(12.5)$ & $45.9(12.5)$ & $45.8(12.5)$ & 0.88 \\
\hline Injury duration (days) & $\begin{array}{l}380.7(1137.3) \\
\text { Median }=112\end{array}$ & $\begin{array}{l}376.4(1145.1) \\
\text { Median }=108\end{array}$ & $\begin{array}{l}392.1(1116.3) \\
\text { Median }=123\end{array}$ & 0.68 \\
\hline Annual Salary $(\$ 10,000 \mathrm{CDN})$ & $49.0(36.4)$ & $48.0(36.1)$ & $51.5(36.9)$ & $0.004 *$ \\
\hline Sex & & & & 0.54 \\
\hline Male & $2691(59.6 \%)$ & $1953(59.3 \%)$ & $738(60.3 \%)$ & \\
\hline Female & $1825(40.4 \%)$ & $1340(40.7 \%)$ & $485(39.7 \%)$ & \\
\hline Occupational category & & & & $0.03 *$ \\
\hline Management & $155(3.4 \%)$ & $107(3.2 \%)$ & $48(3.9 \%)$ & \\
\hline Business, finance, administration & $276(6.1 \%)$ & $219(6.7 \%)$ & $57(4.7 \%)$ & \\
\hline Sciences and related occupations & $93(2.1 \%)$ & $65(2.0 \%)$ & $28(2.3 \%)$ & \\
\hline Health & $478(10.6 \%)$ & $327(9.9 \%)$ & $151(12.3 \%)$ & \\
\hline Education, law and services & $345(7.6 \%)$ & $251(7.6 \%)$ & $94(7.7 \%)$ & \\
\hline Art, culture, recreation and sport & $32(0.7 \%)$ & $23(0.7 \%)$ & $9(0.7 \%)$ & \\
\hline Sales and service & $852(18.9 \%)$ & $650(19.7 \%)$ & $202(16.5 \%)$ & \\
\hline Trades and transport & $1903(42.1 \%)$ & $1364(41.4 \%)$ & $539(44.1 \%)$ & \\
\hline Production & $128(2.8 \%)$ & $95(2.9 \%)$ & $33(2.7 \%)$ & \\
\hline Manufacturing & $248(5.5 \%)$ & $187(5.7 \%)$ & $61(5.0 \%)$ & \\
\hline Unknown & $6(0.1 \%)$ & $5(0.2 \%)$ & $1(0.1 \%)$ & \\
\hline Employed (\% yes) & $3672(81.3 \%)$ & $2737(83.1 \%)$ & $935(76.5 \%)$ & $<0.001 *$ \\
\hline Currently working (\% yes) & $2193(48.6 \%)$ & $1730(52.5 \%)$ & $463(37.9 \%)$ & $<0.001 *$ \\
\hline Modified work available (\% yes) & $2270(50.3 \%)$ & $1708(51.9 \%)$ & $562(46.0 \%)$ & $<0.001 *$ \\
\hline Diagnosis & & & & 0.08 \\
\hline Sprain/strain & $2160(47.8 \%)$ & $1585(48.1 \%)$ & $575(47.0 \%)$ & \\
\hline Joint disorder & $1084(24.0 \%)$ & $797(24.2 \%)$ & $287(23.5 \%)$ & \\
\hline Fracture & $279(6.2 \%)$ & $188(5.7 \%)$ & $91(7.4 \%)$ & \\
\hline Contusion & $334(7.4 \%)$ & $252(7.7 \%)$ & $82(6.7 \%)$ & \\
\hline Laceration & $55(1.2 \%)$ & $40(1.2 \%)$ & $15(1.2 \%)$ & \\
\hline Dislocation & $113(2.5 \%)$ & $74(2.2 \%)$ & $39(3.2 \%)$ & \\
\hline Nerve damage & $98(2.2 \%)$ & $79(2.4 \%)$ & $19(1.6 \%)$ & \\
\hline Other/unspecified & $393(8.7 \%)$ & $278(8.4 \%)$ & $115(9.4 \%)$ & \\
\hline Part of body & & & & 0.75 \\
\hline Upper extremity & $1760(39.0 \%)$ & $1267(38.5 \%)$ & $493(40.3 \%)$ & \\
\hline Back & $934(20.7 \%)$ & $683(20.7 \%)$ & $251(20.5 \%)$ & \\
\hline Lower extremity & $68(17.0 \%)$ & $5573(17.4 \%)$ & $195(15.9 \%)$ & \\
\hline Neck & $387(8.6 \%)$ & $282(8.6 \%)$ & $105(8.6 \%)$ & \\
\hline Other & $667(14.8 \%)$ & $488(14.8 \%)$ & $179(14.6 \%)$ & \\
\hline Comorbid injury (\% yes) & $1656(36.7 \%)$ & $1214(36.9 \%)$ & $442(36.1 \%)$ & 0.68 \\
\hline \multicolumn{5}{|l|}{ Type of work assessment conducted } \\
\hline Basic (1-day) & $4140(91.7 \%)$ & $2935(89.1 \%)$ & $1205(98.5 \%)$ & $<0.001 *$ \\
\hline Comprehensive (2-day) & $376(8.3 \%)$ & $358(10.9 \%)$ & $18(1.5 \%)$ & \\
\hline Language interpreter required (\% yes) & $111(2.5 \%)$ & $92(2.8 \%)$ & $19(1.6 \%)$ & $0.02 *$ \\
\hline
\end{tabular}

$F C E$ functional capacity evaluation, FIT functional interview tool 


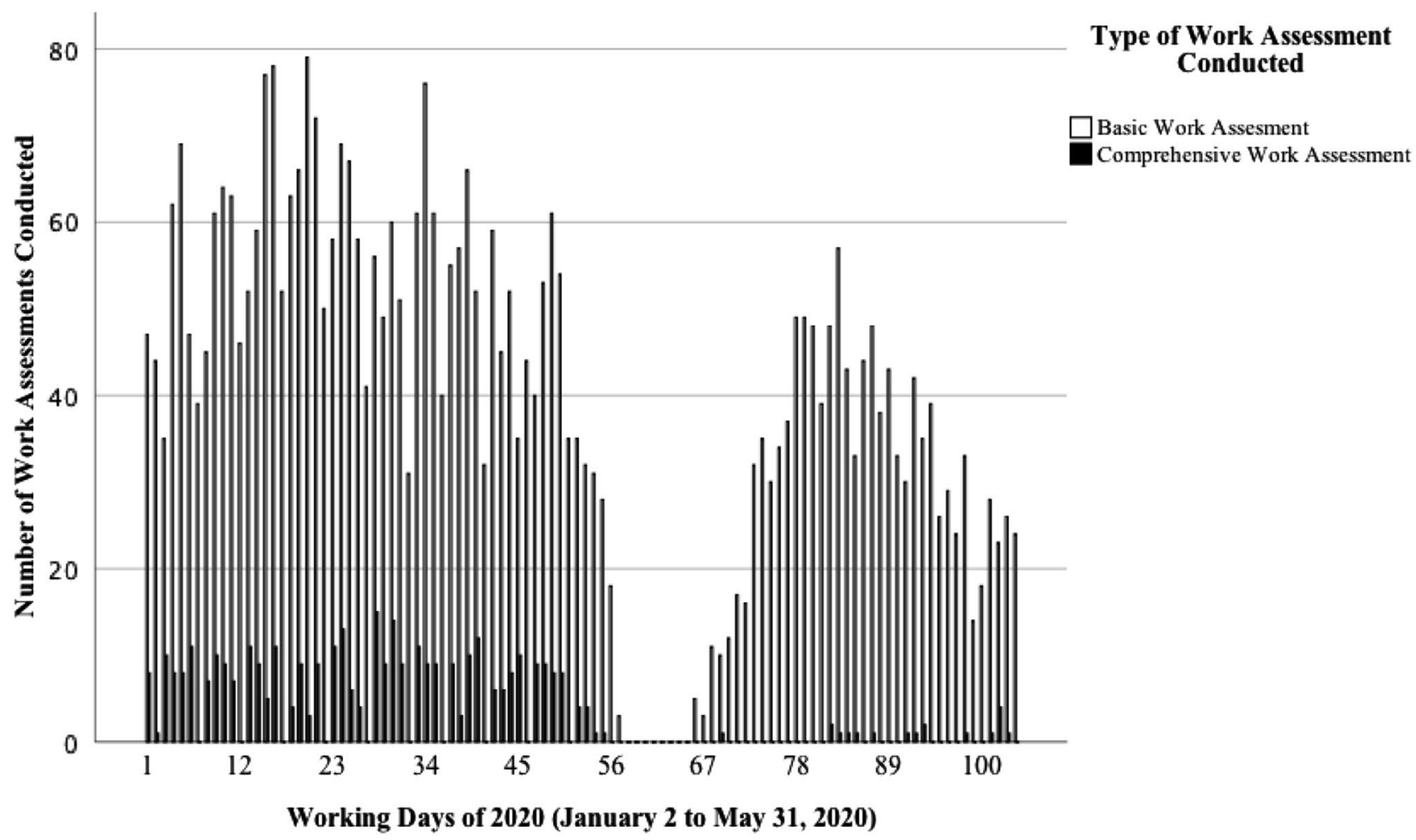

Fig. 1 Bar Graph Showing Number of Work Assessments Conducted with Albertan Injured Workers Each Day of 2020 (January 2-May 31). Mean assessments per week January 1 to March 23,

rehabilitation before and after the transition to remote services are shown in Table 4 . The majority (58.5\%) underwent Functional Restoration programs, with a significantly larger percentage $(67.1 \%$ versus $57.1 \%)$ of functional restoration programs observed after transition to remote services. Worker characteristics were similar before and after transitioning to remote services, however those undergoing remote programs were less likely to require an interpreter $(0.8 \%$ versus $3.6 \%)$ and less likely to have modified work available $(50.6 \%$ versus $64.5 \%)$.

\section{Adverse Events}

No formal complaints were received or adverse events reported within the first two months of remote rehabilitation service delivery. No complaints related to security or privacy during telerehabilitation were received. Informally, some questions and concerns from case managers were received about the FIT assessment protocol. Some expressed concerns about the FIT resulting in lower functional levels than earlier FCE results, with at least one permanent modified job offer from an employer compromised as a result. Clinicians voiced mixed opinions about using remote service delivery.
$2020=244.6($ SD 83.5). Mean assessments per week April 3 to May $31,2020=135.9($ SD 74.5$)$

\section{Discussion}

The unique circumstances created by the COVID-19 pandemic and restrictions put in place to reduce spread of the virus warrant research to document and explore the transition to remote delivery of health care services. It appears that widescale remote occupational rehabilitation services in this Canadian compensation context has been successful, with few adverse effects or complaints reported despite hundreds of injured workers undergoing work assessment and rehabilitation. However, the jurisdiction appears to have taken a cautious approach with few complex cases referred for comprehensive work assessment or rehabilitation. Overall work assessment and rehabilitation program volumes also reduced after the switch to remote services. These findings provide important understanding of remote services for injured workers and inform global efforts to continue offering rehabilitation services while remaining physically distant due to the COVID-19 pandemic.

Further research is needed to follow these workers for a longer duration, ideally at least one year. While a previous trial using the FIT protocol found little difference in long-term outcomes when compared to traditional performance-based assessment, the trial was still conducted 
Table 2 Outcome recommendations following basic work assessments before and after transitioning to remote services

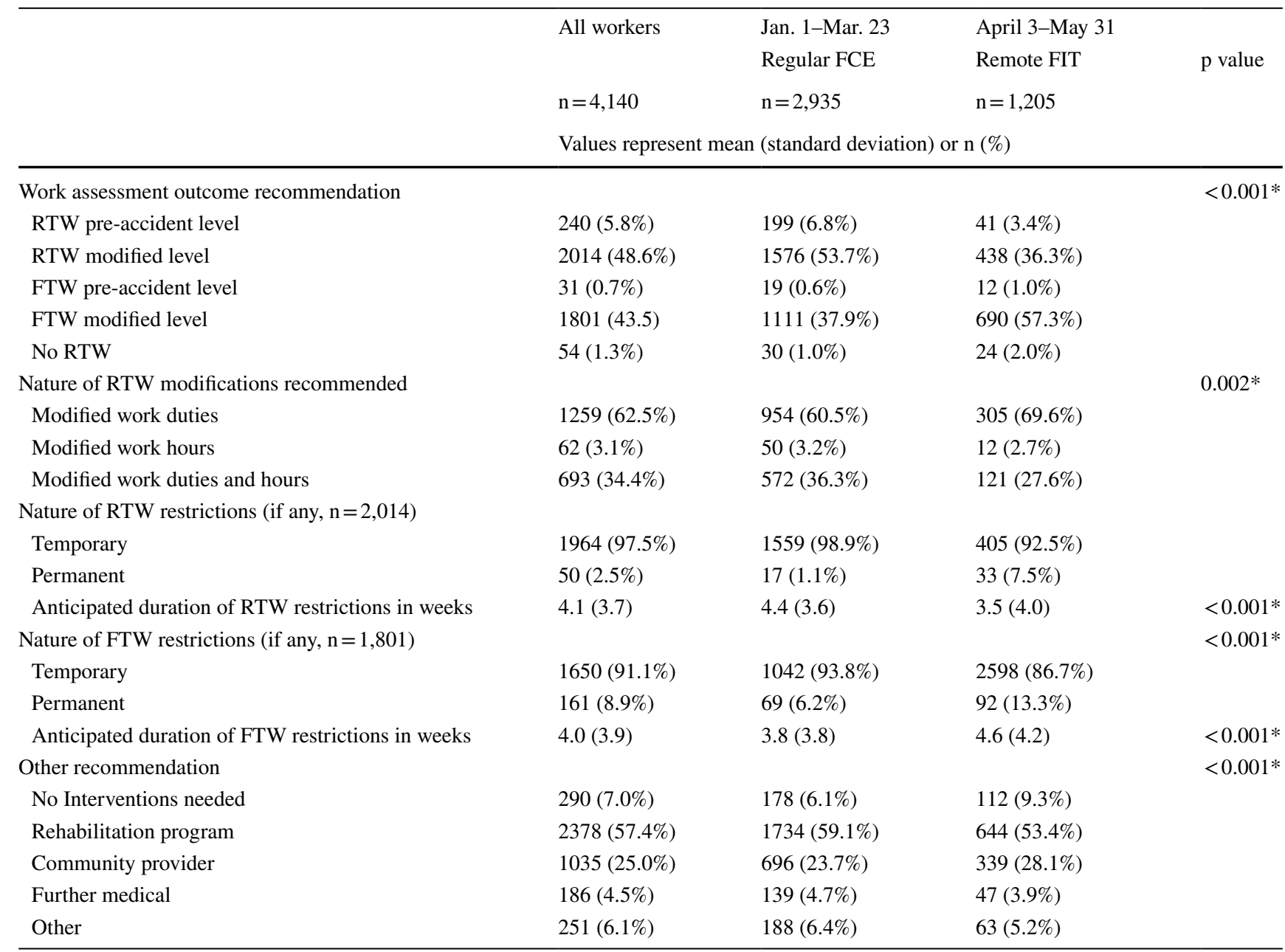

$F C E$ functional capacity evaluation, FIT functional interview tool, $R T W$ return to work, $F T W$ fit to work

*Indicates statistically significant difference between injured workers underdoing regular versus remote work assessment on independent $\mathrm{t}$ or chi square tests $(\mathrm{p}<0.05)$

with interviews done in a face-to-face setting. Conducting work assessment or rehabilitation over videoconferencing or telephone is entirely different, and warrants longterm evaluation. Potentially, return to work determinations made using remote technology are not as accurate and lead to delayed or less sustainable work outcomes. Our findings indicate that clinicians conducting remote work assessments made more cautious recommendations, with fewer recommendations for immediate return to preaccident work and a higher percentage of workers given permanent work restrictions. This appeared to cause concern for some case managers, with at least one worker having a permanent job offer compromised because work ability levels determined using the FIT were lower than earlier FCE results. Previous research has indicated that self-reported functional levels are often lower than results of performance-based functional tests, which may explain the observation of fewer recommendations for immediate return to work. However, we did not have access to raw data on individual items within the functional tests conducted to explore this further.

Injured workers undergoing work assessment after the start of the pandemic likely had limited opportunities for returning to work, since within weeks of the public health interventions taking effect unemployment rates in Alberta and Canada more broadly had increased dramatically. This likely affected reasons for referral, clinician decisions, and will impact longer-term outcomes. We observed that more workers referred for remote work assessment (post-COVID) were unemployed, not currently working, and did not have modified work available. There were also significant differences in the availability of modified work among those undergoing rehabilitation. While this may be due to different referral patterns by case managers, it most likely reflects the broader social and economic impact of the pandemic. These broader socioeconomic changes will also impact claim, 
Table 3 Clinical characteristics of injured workers completing self-report questionnaires during basic work assessment

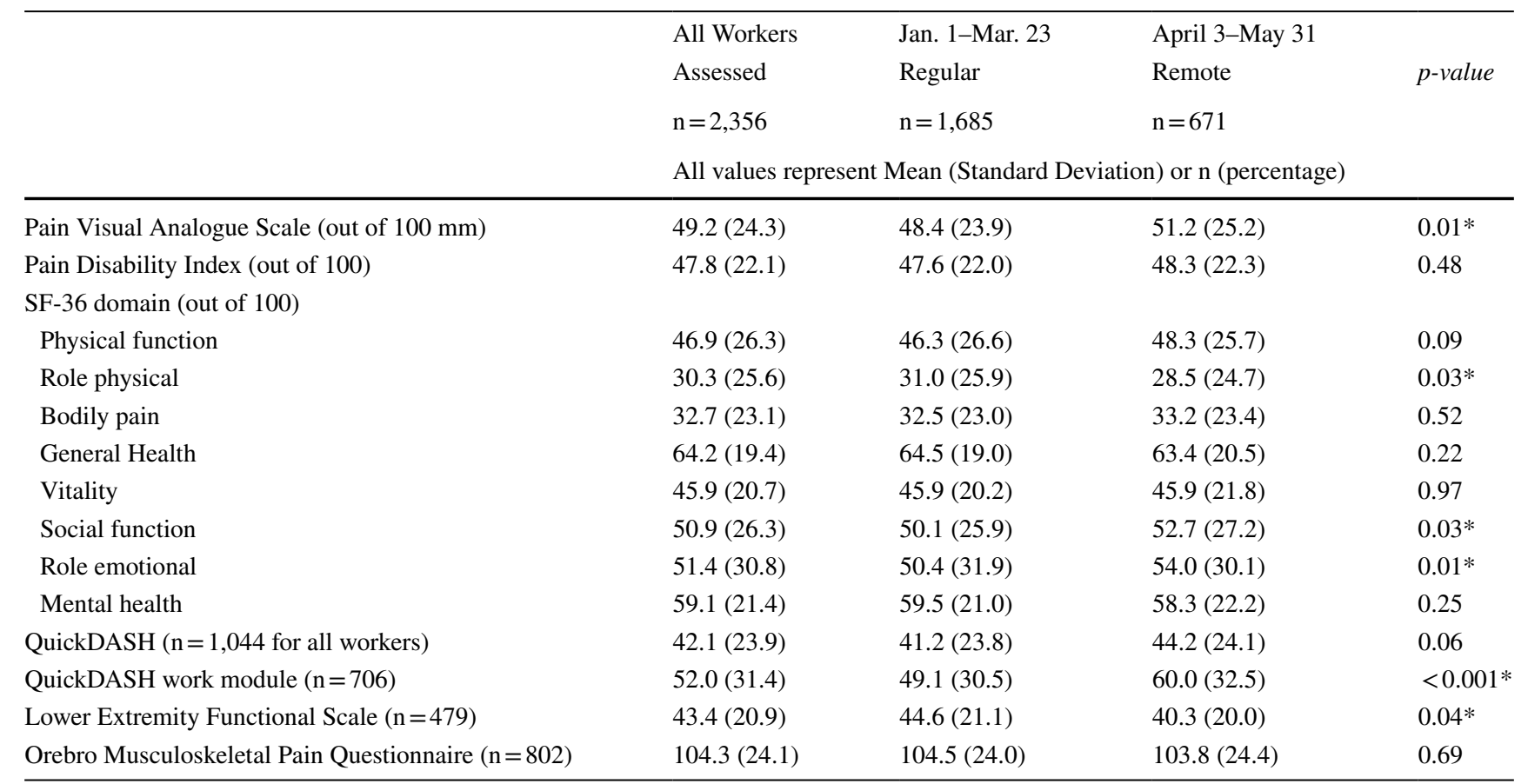

${ }^{*}$ Indicates statistically significant difference between injured workers underdoing regular versus remote work assessment on independent $\mathrm{t}$ or chi square test $(\mathrm{p}<0.05)$

return to work, and rehabilitation outcomes and should be further evaluated.

The transition to telerehabilitation due to the pandemic has implications on traditionally underserved communities and populations. This includes individuals who reside or work in rural or remote areas as well as indigenous communities who often do not have ready access to in-person rehabilitation services [21-23]. The use of telerehabilitation may be an effective option, allowing provision of services to these groups even after the pandemic to help overcome inequities in access to rehabilitation services. Unfortunately, our dataset did not contain information on geographic location or race to explore results in these sub-populations. Future research is needed to examine the effectiveness of telerehabilitation among these groups.

\section{Limitations}

Since this was a descriptive study of early experiences with remote services, the workers were not followed up for any period of time. Long-term outcomes are unknown at this point and these workers should be followed up to determine longer-term sustainable return-to-work outcomes following remote work assessment as well as any subsequent rehabilitation programs. This study was also limited by its use of archived data from WCB-Alberta, which led to a large amount of missing data on self-reported outcome measures. We also did not have access to raw data on the functional scores on the work assessment protocols, which are not consistently collected or available in the database. Further research is needed to rigorously evaluate stakeholder (i.e. worker, employer, clinician, and case manager) perceptions of remote services and satisfaction with care.

\section{Conclusions}

We describe the transition to remote delivery of occupational rehabilitation services due to COVID-19 physical distancing restrictions in one Canadian compensation jurisdiction. It appears the use of remote services was successful but proceeded cautiously, with fewer complex cases being referred for assessment or rehabilitation. Further research examining longer-term work outcomes and stakeholder perceptions should be conducted given the uncertainty around the effectiveness of remote rehabilitation service delivery. 
Table 4 Characteristics of injured workers undergoing occupational rehabilitation programs before and after transitioning to remote services

\begin{tabular}{|c|c|c|c|c|}
\hline & $\begin{array}{l}\text { All workers } \\
\mathrm{n}=1,755\end{array}$ & $\begin{array}{l}\text { In-person program } \\
\mathrm{n}=1,506\end{array}$ & $\begin{array}{l}\text { Remote program } \\
\mathrm{n}=249\end{array}$ & $\mathrm{p}$ value \\
\hline & \multicolumn{4}{|c|}{ Values represent mean (standard deviation) or $\mathrm{n}(\%)$} \\
\hline Type of program & & & & $0.003^{*}$ \\
\hline Functional restoration (FR) & $1027(58.5 \%)$ & $860(57.1 \%)$ & $167(67.1 \%)$ & \\
\hline Hybrid FR and workplace & $479(27.3 \%)$ & $413(27.4 \%)$ & $66(26.5 \%)$ & \\
\hline Complex chronic pain & $123(7.0 \%)$ & $113(7.5 \%)$ & $10(4.0 \%)$ & \\
\hline Brain injury & $71(4.0 \%)$ & $49(4.6 \%)$ & $2(0.8 \%)$ & \\
\hline Cumulative psychological injury & $15(0.9 \%)$ & $15(1.0 \%)$ & $0(0 \%)$ & \\
\hline Traumatic psychological injury & $40(2.3 \%)$ & $36(2.4 \%)$ & $4(1.6 \%)$ & \\
\hline Injury duration (days) & $380.7(1137.3)$ & $243.8(649.3)$ & $213.2(292.2)$ & 0.46 \\
\hline Referral to admission (days) & $6.4(9.9)$ & $6.2(12.7)$ & 0.80 & \\
\hline Age of worker (years) & $45.9(12.5)$ & $45.1(12.2)$ & $44.6(12.6)$ & 0.52 \\
\hline Annual salary $(\$ 10,000 \mathrm{CDN})$ & $48.9(34.3)$ & $45.8(33.0)$ & 0.19 & \\
\hline Sex & & & & 0.10 \\
\hline Male & $993(56.6 \%)$ & $864(57.4 \%)$ & $129(51.8 \%)$ & \\
\hline Female & $762(43.4 \%)$ & $642(42.6 \%)$ & $120(48.2 \%)$ & \\
\hline Language interpreter required (\% yes) & $56(3.2 \%)$ & $54(3.6 \%)$ & $2(0.8 \%)$ & $0.02 *$ \\
\hline Employed (\% yes) & $1477(84.2 \%)$ & $1273(84.5 \%)$ & $204(81.9 \%)$ & 0.30 \\
\hline Currently working (\% yes) & $1021(58.2 \%)$ & $889(59.0 \%)$ & $132(53.0 \%)$ & 0.08 \\
\hline Modified work available ( $\%$ yes) & $1098(62.6 \%)$ & $972(64.5 \%)$ & $126(50.6 \%)$ & $<0.001 *$ \\
\hline Length of program & & & & 0.004 \\
\hline 1 to 19 days & $665(37.9 \%)$ & $550(36.5 \%)$ & $115(46.2 \%)$ & \\
\hline $20+$ days & $1090(62.1 \%)$ & $956(63.5 \%)$ & $134(53.8 \%)$ & \\
\hline Discharge outcome & & & & 0.20 \\
\hline Return to work & $1139(64.9 \%)$ & $990(65.7 \%)$ & $149(59.8 \%)$ & \\
\hline Fit to work & $592(33.7 \%)$ & $496(32.9 \%)$ & $96(38.6 \%)$ & \\
\hline No return to work & $24(1.4 \%)$ & $20(1.3 \%)$ & $4(1.6 \%)$ & \\
\hline
\end{tabular}

Acknowledgements No funding was received in support of this research. Data were provided by the Workers' Compensation Board of Alberta.

Funding No funding was received for this research.

\section{Compliance with Ethical Standards}

Conflict of interest Douglas Gross, Alexander Asante, Joanne Pawluk, and Riikka Niemeläinen declare that they have no conflict of interest.

Ethical Approval All procedures performed in studies involving human participants were in accordance with the ethical standards of the institutional and/or national research committee and with the 1964 Helsinki declaration and its later amendments or comparable ethical standards.

Informed Consent This study was an analysis of an administrative dataset therefore informed consent for the study was not obtained.

\section{References}

1. Government of Canada Coronavirus Disease (Covid-19) website. Health Canada. 2020. https://www.canada.ca/en/public-health/ services/diseases/coronavirus-disease-covid-19.html. Accessed 2 Sept 2020.

2. Landry MD, Tupetz A, Jalovic D, Sheppard P, Jesus TS, Raman SR. The novel coronavirus (COVID-19): making a connection between infectious disease outbreaks and rehabilitation. Physiotherapy Canada. 2020;e20200019(advance online article):1-3.

3. Eccleston C, Blyth FM, Dear BF, Fisher EA, Keefe FJ, Lynch $\mathrm{ME}$, et al. Managing patients with chronic pain during the COVID-19 outbreak: considerations for the rapid introduction of remotely supported (eHealth) pain management services. Pain. 2020;161(5):889-893. https://doi.org/10.1097/j.pain.0000000000 001885 .

4. Grona SL, Bath B, Busch A, Rotter T, Trask C, Harrison E. Use of videoconferencing for physical therapy in people with musculoskeletal conditions: a systematic review. J Telemed Telecare. 2018;24(5):341-355. https://doi.org/10.1177/1357633X1770078 1.

5. Cottrell MA, Galea OA, O'Leary SP, Hill AJ, Russell TG. Realtime telerehabilitation for the treatment of musculoskeletal conditions is effective and comparable to standard practice: a systematic review and meta-analysis. Clin Rehabil. 2017;31(5):625-638. https://doi.org/10.1177/0269215516645148.

6. Asante AK, Brintnell ES, Gross DP. Functional self-efficacy beliefs influence functional capacity evaluation. J Occup Rehabil. 2007;17(1):73-82. https://doi.org/10.1007/s10926-007-9068-1.

7. Gross DP, Asante AK, Miciak M, Battie MC, Carroll LJ, Sun A, et al. A cluster randomized clinical trial comparing functional 
capacity evaluation and functional interviewing as components of occupational rehabilitation programs. J Occup Rehabil. 2014;24(4):617-630. https://doi.org/10.1007/s10926-013-9491-4.

8. Gross DP, Asante AK, Miciak M, Battie MC, Carroll LJ, Sun $\mathrm{A}$, et al. Are performance-based functional assessments superior to semistructured interviews for enhancing return-to-work outcomes? Arch Phys Med Rehabil. 2014;95(5):807-815.

9. Park J, Gross DP, Rayani F, Norris CM, Roberts MR, James C, et al. Model of human occupation as a framework for implementation of motivational interviewing in occupational rehabilitation. Work. 2019;62(4):629-641. https://doi.org/10.3233/WOR-19289 5.

10. Gross DP, Park J, Rayani F, Norris CM, Esmail S. Motivational interviewing improves sustainable return to work in injured workers after rehabilitation: a cluster randomized controlled trial. Arch Phys Med Rehabil. 2017;98(12):2355-2363. https://doi. org/10.1016/j.apmr.2017.06.003.

11. Park J, Esmail S, Rayani F, Norris CM, Gross DP. Motivational interviewing for workers with disabling musculoskeletal disorders: results of a cluster randomized control trial. J Occup Rehabil. 2018;28(2):252-264. https://doi.org/10.1007/s10926-017-9712-3.

12. Pollard CA. Preliminary validity study of the pain disability index. Percept Mot Skills. 1984;59(3):974.

13. Finch E, Brooks D, Stratford P, Mayo N. Physical rehabilitation outcome measures: a guide to enhanced clinical decision making. 2nd ed. Toronto: Canadian Physiotherapy Association; 2002.

14. McHorney CA, Ware JE, Jr., Raczek AE. The MOS 36-item shortform health survey (SF-36): II. Psychometric and clinical tests of validity in measuring physical and mental health constructs. Med Care. 1993;31(3):247-263.

15. Fan ZJ, Smith CK, Silverstein BA. Assessing validity of the QuickDASH and SF-12 as surveillance tools among workers with neck or upper extremity musculoskeletal disorders. J Hand Ther. 2008;21(4):354-365.

16. Binkley JM, Stratford PW, Lott SA, Riddle DL. The lower extremity functional scale (LEFS): scale development, measurement properties, and clinical application. Phys Ther. 1999;79(4):371-383.
17. Linton SJ, Boersma K. Early identification of patients at risk of developing a persistent back problem: the predictive validity of the orebro musculoskeletal pain questionnaire. Clin J Pain. 2003;19(2):80-86.

18. Margison DA, French DJ. Predicting treatment failure in the subacute injury phase using the orebro musculoskeletal pain questionnaire: an observational prospective study in a workers' compensation system. J Occup Environ Med. 2007;49(1):59-67. https://doi. org/10.1097/JOM.0b013e31802db51e.

19. De Baets S, Calders P, Schalley N, Vermeulen K, Vertriest S, Van Peteghem L, et al. Updating the evidence on functional capacity evaluation methods: a systematic review. J Occup Rehabil. 2018;28(3):418-428. https://doi.org/10.1007/s10926-017-9734-x.

20. Gross DP, Steenstra IA, Shaw W, Yousefi P, Bellinger C, Zaiane $\mathrm{O}$. Validity of the work assessment triage tool for selecting rehabilitation interventions for workers' compensation claimants with musculoskeletal conditions. J Occup Rehabil. 2020;30(3):318330. https://doi.org/10.1007/s10926-019-09843-4.

21. Lovo S, Harrison L, O'Connell ME, Trask C, Bath B. Experience of patients and practitioners with a team and technology approach to chronic back disorder management. J Multidiscip Healthc. 2019;12:855-869. https://doi.org/10.2147/JMDH.S208888.

22. Macpherson RA, Amick BC 3rd, Collie A, Hogg-Johnson S, Koehoorn M, Smith PM, et al. Urban-rural differences in the duration of injury-related work disability in six Canadian provinces. J Occup Environ Med. 2020;62(5):e200-e207. https://doi. org/10.1097/JOM.0000000000001850.

23. Nader F, Kolahdooz F, Sharma S. Assessing health care access and use among indigenous peoples in Alberta: a systematic review. J Health Care Poor Underserved. 2017;28(4):1286-1303. https://doi.org/10.1353/hpu.2017.0114.

Publisher's Note Springer Nature remains neutral with regard to jurisdictional claims in published maps and institutional affiliations. 